\title{
Generalized Deformed Commutation Relations with Nonzero Minimal Uncertainties in Position and/or Momentum and Applications to Quantum Mechanics
}

\author{
Christiane QUESNE ${ }^{\dagger}$ and Volodymyr M. TKACHUK ${ }^{\ddagger}$ \\ † Physique Nucléaire Théorique et Physique Mathématique, Université Libre de Bruxelles, \\ Campus de la Plaine CP229, Boulevard du Triomphe, B-1050 Brussels, Belgium \\ E-mail: cquesne@ulb.ac.be \\ ¥ Ivan Franko Lviv National University, Chair of Theoretical Physics, \\ 12 Drahomanov Str., Lviv UA-79005, Ukraine \\ E-mail: tkachuk@ktf.franko.lviv.ua
}

Received November 22, 2006; Published online January 31, 2007

Original article is available at http://www.emis.de/journals/SIGMA/2007/016/

\begin{abstract}
Two generalizations of Kempf's quadratic canonical commutation relation in one dimension are considered. The first one is the most general quadratic commutation relation. The corresponding nonzero minimal uncertainties in position and momentum are determined and the effect on the energy spectrum and eigenfunctions of the harmonic oscillator in an electric field is studied. The second extension is a function-dependent generalization of the simplest quadratic commutation relation with only a nonzero minimal uncertainty in position. Such an uncertainty now becomes dependent on the average position. With each function-dependent commutation relation we associate a family of potentials whose spectrum can be exactly determined through supersymmetric quantum mechanical and shape invariance techniques. Some representations of the generalized Heisenberg algebras are proposed in terms of conventional position and momentum operators $x, p$. The resulting Hamiltonians contain a contribution proportional to $p^{4}$ and their $p$-dependent terms may also be functions of $x$. The theory is illustrated by considering Pöschl-Teller and Morse potentials.
\end{abstract}

Key words: deformed algebras; uncertainty relations; supersymmetric quantum mechanics; shape invariance

2000 Mathematics Subject Classification: 37N20; 81R15

\section{Introduction}

During recent years, there has been much interest in studying theories characterized by a minimal observable length $\Delta X_{0}$. Some works $[1,2]$ in the context of perturbative string theory have indeed suggested a generalized uncertainty principle, which, for dimensionless operators, reads

$$
\Delta X \geq \frac{1}{2}\left(\frac{1}{\Delta P}+\beta \Delta P\right),
$$

where $\beta$ is some very small positive parameter. Equation (1.1) leads to a nonzero minimal uncertainty in position (or minimal length) $\Delta X_{0}=\sqrt{\beta}$. Another consequence of (1.1) is a mixing between UV and IR divergences. Both properties have also emerged from many other studies in string theory and quantum gravity (see, e.g., [3, 4, 5, 6] and references quoted therein). 
The generalized uncertainty principle (1.1) implies some modification of the canonical commutation relations. Amongst several possibilities, one of the most promising and simplest proposal [2]

$$
[X, P]=\mathrm{i}\left(1+\beta P^{2}\right),
$$

is based on the addition of some small quadratic correction. This line of approach has been thoroughly investigated by Kempf [7, 8, 9, 10], who considered more general quadratic corrections in $D$ dimensions and also suggested that the absence of plane waves or momentum eigenvectors on generic curved spaces would give rise to a minimal observable momentum $\Delta P_{0}$ [11].

Since the UV/IR mixing allows one to probe high-energy physics by low-energy one, it justifies the use of quantum mechanics in the presence of a minimal length. Investigating the influence of the minimal length assumption on the energy spectrum of quantum systems has become an interesting issue primarily for two reasons. First, this may help to set some upper bounds on the value of the minimal length. In this connection, one may quote some studies of the hydrogen atom $[12,13,14]$, of the problem of a particle in a gravitational quantum well [15], and of the application of the one-dimensional Dirac oscillator to quark-gluon plasmas [16]. Furthermore, the classical limit has also provided some interesting insights into some cosmological problems $[17,18]$. Second, it has been argued [10] that quantum mechanics with a minimal length may also be useful to describe non-pointlike particles, such as quasiparticles and various collective excitations in solids, or composite particles, e.g., hadrons. The formalism then provides us with an effective theory of such systems in terms of a parameter $\Delta X_{0}$ related to the finite size of the particles.

Solving quantum mechanical problems with deformed canonical commutation relations, however, usually turns out to be much more difficult than with conventional ones. This is indeed the case when employing Kempf's quadratic commutation relations, so that only a few examples have been considered in such a context.

In the simplest case where one considers a nonzero minimal uncertainty in position only, several quantum mechanical systems have been successfully dealt with by solving the corresponding Schrödinger equation in momentum space. Such problems include the one- and $D$-dimensional harmonic oscillators $[9,19]$, for which exact solutions have been provided, the hydrogen atom, for which perturbative $[12,14]$, numerical [13], or one-dimensional exact [20] results have been obtained, and the gravitational quantum well [15], which has been treated perturbatively. The use of WKB approximation has also been tested [21].

In the more complicated case where one considers nonzero minimal uncertainties in both position and momentum and one can therefore only resort to a generalized Fock space (or corresponding Bargmann) representation [7, 8, 22], we have recently proposed an entirely different approach to the one-dimensional harmonic oscillator problem [23]. It is based upon an extension to the deformed commutation relation in hand of supersymmetric quantum mechanical (SUSYQM) techniques [24]. When supplemented with shape invariance (SI) under parameter translation [25, 26] or parameter scaling [27, 28, 29, 30, 31, 32], these are known to provide a very powerful method for exactly solving problems in standard quantum mechanics. This is the case here too, since they have also allowed us [33] to deal with the one-dimensional harmonic oscillator in a uniform electric field and to algebraically rederive the results of $[9,19]$.

The only relativistic problem that has been exactly solved so far in a deformed space with minimal length is the Dirac oscillator, either in three dimensions using our extended SUSYQM and SI method [34], or in one dimension by directly solving the differential equation in momentum representation [16].

The purpose of the present paper is twofold: first, to propose some generalizations of Kempf's quadratically-deformed canonical commutation relation in one dimension and to analyze how such extensions affect the uncertainties in position and momentum, and second, to further 
illustrate the power of combined SUSYQM and SI techniques in solving some eigenvalue problems corresponding to these new deformed commutation relations.

At this stage, it should be stressed that our work is mainly aimed at applications to systems of non-pointlike particles, especially in condensed-matter physics, where one-dimensional systems may be experimentally produced. However, our ultimate goal would be to extend the simple models presented here to more than one dimension, in which case the noncommutativity of position coordinates (and possibly also that of momentum ones) would become of utmost importance.

The paper is organized as follows. The most general quadratic canonical commutation relation with nonzero minimal uncertainties in both position and momentum is considered in Section 2. Then a function-dependent generalization of the quadratic commutation relation with only a nonzero minimal uncertainty in position is reviewed in Section 3. Finally, in Section 4, we summarize our results and sketch some of their possible applications to a variety of problems.

\section{Generalized quadratic commutation relation}

\subsection{Commutation relation and uncertainty relation}

The most general quadratically-deformed canonical commutation relation can be written as

$$
[X, P]=\mathrm{i}\left(1+\alpha X^{2}+\beta P^{2}+\kappa X P+\kappa^{*} P X\right),
$$

where $\alpha, \beta \in \mathbb{R}$ and $\kappa \in \mathbb{C}$ are assumed to be very small parameters (i.e., $|\alpha|,|\beta|,|\kappa| \ll 1$ ), while $X$ and $P$ are dimensionless operators. Throughout this paper, we use units wherein $\hbar=1$ (as well as $\ell=m=1$, where $\ell$ and $m$ denote the characteristic length and the particle mass for the quantum mechanical system under consideration).

Equation (2.1) admits two important special cases: choosing $\kappa=0$ and $\alpha, \beta>0$ leads to Kempf's commutation relation characterized by nonzero minimal uncertainties $\Delta X_{0}=$ $\sqrt{\beta /(1-\alpha \beta)}$ and $\Delta P_{0}={\sqrt{\alpha /(1-\alpha \beta)^{1}}}^{1}$, whereas selecting $\alpha=\beta=0$ gives rise to the $q$ deformed Heisenberg algebra $q X P-q^{*} P X=\mathrm{i}$ with $q \equiv 1-\mathrm{i} \kappa[35]$.

In the general case, on setting $\kappa=\kappa_{1}+\mathrm{i} \kappa_{2}, \kappa_{1}, \kappa_{2} \in \mathbb{R}$, equation (2.1) may be rewritten as

$$
[\tilde{X}, \tilde{P}]=\mathrm{i}\left[1+\tilde{\alpha} \tilde{X}^{2}+\tilde{\beta} \tilde{P}^{2}+\tilde{\kappa}_{1}(\tilde{X} \tilde{P}+\tilde{P} \tilde{X})\right]
$$

in terms of some rescaled operators and parameters, $\tilde{X}=X \sqrt{1+\kappa_{2}}, \tilde{P}=P \sqrt{1+\kappa_{2}}, \tilde{\alpha}=$ $\alpha /\left(1+\kappa_{2}\right), \tilde{\beta}=\beta /\left(1+\kappa_{2}\right)$ and $\tilde{\kappa}_{1}=\kappa_{1} /\left(1+\kappa_{2}\right)$. This simple property allows us to restrict ourselves to real values of $\kappa$. Hence, in the remainder of this section, we will inquire into the influence of an additional term i $\kappa(X P+P X)$, with $\kappa \in \mathbb{R}$, on the right-hand side of Kempf's deformed commutation relation $[X, P]=\mathrm{i}\left(1+\alpha X^{2}+\beta P^{2}\right)$ with $\alpha, \beta \in \mathbb{R}^{+}$.

On performing the rotation

$$
X=X^{\prime} \cos \varphi+P^{\prime} \sin \varphi, \quad P=-X^{\prime} \sin \varphi+P^{\prime} \cos \varphi,
$$

such a generalized deformed commutation relation can be rewritten as

$$
\left[X^{\prime}, P^{\prime}\right]=\mathrm{i}\left[1+\alpha^{\prime} X^{\prime 2}+\beta^{\prime} P^{\prime 2}+\kappa^{\prime}\left(X^{\prime} P^{\prime}+P^{\prime} X^{\prime}\right)\right]
$$

where

$$
\alpha^{\prime}=\frac{1}{2}(\alpha+\beta)+\frac{1}{2}(\alpha-\beta) \cos 2 \varphi-\kappa \sin 2 \varphi
$$

\footnotetext{
${ }^{1}$ It should be stressed that the positivity of $\alpha$ and $\beta$ is essential to get nonvanishing values for $\Delta X_{0}$ and $\Delta P_{0}$.
} 


$$
\begin{aligned}
\beta^{\prime} & =\frac{1}{2}(\alpha+\beta)-\frac{1}{2}(\alpha-\beta) \cos 2 \varphi+\kappa \sin 2 \varphi \\
\kappa^{\prime} & =\frac{1}{2}(\alpha-\beta) \sin 2 \varphi+\kappa \cos 2 \varphi
\end{aligned}
$$

Equation (2.3) can be simplified by choosing $\kappa^{\prime}=0$. Here we have to distinguish between $\alpha \neq \beta$ and $\alpha=\beta$. In the former case

$$
\tan 2 \varphi=-\frac{2 \kappa}{\alpha-\beta}
$$

where we may restrict $\varphi$ to the interval $-\pi / 4<\varphi<\pi / 4$. As a consequence

$$
\cos 2 \varphi=\frac{|\alpha-\beta|}{\delta}, \quad \sin 2 \varphi=-\frac{2 \sigma \kappa}{\delta}, \quad \delta \equiv \sqrt{(\alpha-\beta)^{2}+4 \kappa^{2}}, \quad \sigma \equiv \frac{(\alpha-\beta)}{|\alpha-\beta|} .
$$

In the latter case, $\cos 2 \varphi=0$ may be achieved by choosing $\varphi=\pi / 4$, so that

$$
\alpha^{\prime}=\alpha-\kappa, \quad \beta^{\prime}=\beta+\kappa .
$$

With such choices for $\varphi$, the transformed operators $X^{\prime}, P^{\prime}$ satisfy a commutation relation similar to that of Kempf provided $\alpha^{\prime}$ and $\beta^{\prime}$ are positive. From (2.4), this amounts to the two conditions

$$
\alpha+\beta>0, \quad\left|\frac{1}{2}(\alpha-\beta) \cos 2 \varphi-\kappa \sin 2 \varphi\right|<\frac{1}{2}(\alpha+\beta) .
$$

If $\alpha \neq \beta$, equation (2.5) allows us to transform the second condition into $\kappa^{2}<\alpha \beta$, which can only be valid if $\alpha \beta>0$. On combining the results, we are led to the restrictions

$$
\alpha>0, \quad \beta>0, \quad|\kappa|<\sqrt{\alpha \beta} .
$$

It is straightforward to see that equation (2.7) remains true for $\alpha=\beta$. We therefore conclude that the generalized commutation relation (2.1) with $\kappa \in \mathbb{R}$ is equivalent to Kempf's one up to a rotation of angle $-\pi / 4<\varphi \leq \pi / 4$ if and only if equation (2.7) is fulfilled.

We now plan to find the minimal uncertainties $\Delta X_{0}$ and $\Delta P_{0}$ corresponding to (2.1), (2.7), and generalizing those obtained by Kempf.

The uncertainty relation associated with (2.1) for real values of $\kappa$ can be written as

$$
\Delta X \Delta P \geq \frac{1}{2}\left|1+\gamma+\alpha(\Delta X)^{2}+\beta(\Delta P)^{2}+\kappa\langle\hat{X} \hat{P}+\hat{P} \hat{X}\rangle\right|
$$

where $\hat{X} \equiv X-\langle X\rangle, \hat{P} \equiv P-\langle P\rangle$, and $\gamma \equiv \alpha\langle X\rangle^{2}+\beta\langle P\rangle^{2}+2 \kappa\langle X\rangle\langle P\rangle$. From the inequality $|\langle A B+B A\rangle| \leq 2 \sqrt{\left\langle A^{2}\right\rangle\left\langle B^{2}\right\rangle}$ valid for any two Hermitian operators $A, B$, it follows that $\mid\langle\hat{X} \hat{P}+$ $\hat{P} \hat{X}\rangle \mid \leq 2 \Delta X \Delta P$. Furthermore, conditions (2.7) imply that $\gamma \geq 0$, hence $1+\gamma+\alpha(\Delta X)^{2}+$ $\beta(\Delta P)^{2}>0$. Equation (2.8) may therefore be transformed into

$$
\Delta X \Delta P \geq \frac{1}{2}\left[1+\gamma+\alpha(\Delta X)^{2}+\beta(\Delta P)^{2}-2|\kappa| \Delta X \Delta P\right]
$$

where we have used property (2.7) again to drop the absolute value on the right-hand side. It is now straightforward to rewrite $(2.9)$ as

$$
\Delta \bar{X} \Delta \bar{P} \geq \frac{1}{2}\left[1+\bar{\alpha}(\Delta \bar{X})^{2}+\bar{\beta}(\Delta \bar{P})^{2}\right]
$$


with

$$
\Delta \bar{X} \equiv \sqrt{\frac{1+|\kappa|}{1+\gamma}} \Delta X, \quad \Delta \bar{P} \equiv \sqrt{\frac{1+|\kappa|}{1+\gamma}} \Delta P, \quad \bar{\alpha} \equiv \frac{\alpha}{1+|\kappa|}, \quad \bar{\beta} \equiv \frac{\beta}{1+|\kappa|} .
$$

Since the last inequality (2.10) is of the same type as that considered by Kempf, we know that there exist nonzero minimal values $\Delta \bar{X}_{0}=\sqrt{\bar{\beta} /(1-\bar{\alpha} \bar{\beta})}, \Delta \bar{P}_{0}=\sqrt{\bar{\alpha} /(1-\bar{\alpha} \bar{\beta})}$ of $\Delta \bar{X}$ and $\Delta \bar{P}$. From this, we infer that there also exist nonzero minimal values of $\Delta X$ and $\Delta P$, given by

$$
\Delta X_{\min }=\sqrt{\frac{\beta(1+\gamma)}{(1+|\kappa|)^{2}-\alpha \beta}}, \quad \Delta P_{\min }=\sqrt{\frac{\alpha(1+\gamma)}{(1+|\kappa|)^{2}-\alpha \beta}},
$$

so that the absolutely smallest uncertainties in $X$ and $P$ are

$$
\Delta X_{0}=\sqrt{\frac{\beta}{(1+|\kappa|)^{2}-\alpha \beta}}, \quad \Delta P_{0}=\sqrt{\frac{\alpha}{(1+|\kappa|)^{2}-\alpha \beta}} .
$$

Had we considered equation (2.1) for complex values of $\kappa$ (and positive values of $\alpha, \beta$ ), equation (2.11) would have been valid for $\Delta \tilde{X}_{0}, \Delta \tilde{P}_{0}, \tilde{\alpha}, \tilde{\beta}$, and $\tilde{\kappa}_{1}$, provided $\left|\tilde{\kappa}_{1}\right|<\sqrt{\tilde{\alpha} \tilde{\beta}}$. Rescaling the operators and parameters, as explained below equation (2.2), would then have led us to minimal uncertainties given by

$$
\Delta X_{0}=\sqrt{\frac{\beta}{\left(1+\kappa_{2}+\left|\kappa_{1}\right|\right)^{2}-\alpha \beta}}, \quad \Delta P_{0}=\sqrt{\frac{\alpha}{\left(1+\kappa_{2}+\left|\kappa_{1}\right|\right)^{2}-\alpha \beta}},
$$

provided $\left|\kappa_{1}\right|<\sqrt{\alpha \beta}$. Observe that setting $\kappa_{1}=\kappa_{2}=0$ in (2.12) gives back Kempf's results, as it should be.

\subsection{Application to the harmonic oscillator in an electric field}

In the present subsection, we plan to study the influence of the $\kappa$-dependent terms in equation (2.1) on the one-dimensional quantum mechanical systems considered in $[23,33]$ under the assumptions that $\kappa \in \mathbb{R}$ and conditions (2.7) are satisfied.

Since the harmonic oscillator Hamiltonian satisfies the relation $H=(1 / 2)\left(P^{2}+X^{2}\right)=$ $(1 / 2)\left(P^{\prime 2}+X^{\prime 2}\right)$, where $X^{\prime}$ and $P^{\prime}$ fulfil equation (2.3) with $\kappa^{\prime}=0$, its spectrum is independent of the presence of $\kappa$-dependent terms in (2.1).

This is not the case, however, for the harmonic oscillator in a uniform electric field $\mathcal{E}$, since its Hamiltonian becomes

$$
H=\frac{1}{2}\left(P^{2}+X^{2}\right)-\mathcal{E} X=\frac{1}{2}\left(P^{\prime 2}+X^{\prime 2}\right)-\mathcal{E}\left(X^{\prime} \cos \varphi+P^{\prime} \sin \varphi\right),
$$

where there is an additional term proportional to $P^{\prime}$. To take care of this change, let us factorize $H$ as

$$
H=B^{+}(g, s, r, \nu) B^{-}(g, s, r, \nu)+\epsilon_{0},
$$

where

$$
B^{ \pm}(g, s, r, \nu)=\frac{1}{\sqrt{2}}\left(\mp \mathrm{i} g P^{\prime}+s X^{\prime}+r \mp \mathrm{i} \nu\right)
$$


with $s, g, r, \nu, \epsilon_{0} \in \mathbb{R}$ and $s, g>0$. Observe that $\nu$ is a new parameter not appearing in [33]. From (2.13)-(2.15), we get

$$
\begin{aligned}
& g=s k, \quad s=\frac{1}{\sqrt{1-\alpha^{\prime} k}}, \quad k=\frac{1}{2}\left(\beta^{\prime}-\alpha^{\prime}+\sqrt{1+\frac{1}{4}\left(\beta^{\prime}-\alpha^{\prime}\right)^{2}}\right), \\
& r=-\frac{\mathcal{E} \cos \varphi}{s}, \quad \nu=-\frac{\mathcal{E} \sin \varphi}{g}, \quad \epsilon_{0}=\frac{1}{2}\left(g s-r^{2}-\nu^{2}\right)
\end{aligned}
$$

in terms of the transformed parameters $\alpha^{\prime}, \beta^{\prime}$, given either in (2.4) and (2.5) or in (2.6).

More generally, $H$ is the first member $H_{0}$ of a hierarchy of Hamiltonians

$$
H_{i}=B^{+}\left(g_{i}, s_{i}, r_{i}, \nu_{i}\right) B^{-}\left(g_{i}, s_{i}, r_{i}, \nu_{i}\right)+\sum_{j=0}^{i} \epsilon_{j}, \quad i=0,1,2, \ldots,
$$

satisfying the SI condition

$$
\begin{aligned}
& B^{-}\left(g_{i}, s_{i}, r_{i}, \nu_{i}\right) B^{+}\left(g_{i}, s_{i}, r_{i}, \nu_{i}\right) \\
& \quad=B^{+}\left(g_{i+1}, s_{i+1}, r_{i+1}, \nu_{i+1}\right) B^{-}\left(g_{i+1}, s_{i+1}, r_{i+1}, \nu_{i+1}\right)+\epsilon_{i+1}
\end{aligned}
$$

where $i=0,1,2, \ldots, g_{0}=g, s_{0}=s, r_{0}=r$, and $\nu_{0}=\nu$. The solution to such a condition is similar to that carried out in [33], so that we only state here the results

$$
\begin{array}{ll}
g_{i}=g q^{i / 2} \frac{1+t q^{-i}}{1+t}, & s_{i}=s q^{i / 2} \frac{1-t q^{-i}}{1-t}, \quad r_{i}=r q^{-i / 2} \frac{1-t}{1-t q^{-i}}, \\
\nu_{i}=\nu q^{-i / 2} \frac{1+t}{1+t q^{-i}}, & \epsilon_{i+1}=\frac{1}{2}\left(g_{i} s_{i}+g_{i+1} s_{i+1}+r_{i}^{2}-r_{i+1}^{2}+\nu_{i}^{2}-\nu_{i+1}^{2}\right),
\end{array}
$$

where

$$
q \equiv \frac{1+\sqrt{\alpha^{\prime} \beta^{\prime}}}{1-\sqrt{\alpha^{\prime} \beta^{\prime}}}, \quad t \equiv \frac{g-\gamma s}{g+\gamma s}, \quad \gamma \equiv \sqrt{\frac{\beta^{\prime}}{\alpha^{\prime}}} .
$$

The energy spectrum of $H$ now reads

$$
E_{n}(\mathcal{E})=\sum_{i=0}^{n} \epsilon_{i}=E_{n}(0)+\Delta E_{n}^{(1)}(\mathcal{E})+\Delta E_{n}^{(2)}(\mathcal{E}),
$$

where $E_{n}(0)$ are the harmonic oscillator Hamiltonian eigenvalues in the absence of electric field (see equation (2.25) of [33]), while

$$
\begin{aligned}
& \Delta E_{n}^{(1)}(\mathcal{E})=-\frac{2 \gamma^{2} \mathcal{E}^{2} \cos ^{2} \varphi}{u^{2}} q^{-n}\left(1-t q^{-n}\right)^{-2}=-\frac{1}{2} K^{2} q^{n} z_{n}^{2}, \\
& \Delta E_{n}^{(2)}(\mathcal{E})=-\frac{2 \mathcal{E}^{2} \sin ^{2} \varphi}{u^{2}} q^{-n}\left(1+t q^{-n}\right)^{-2}=-\frac{1}{2} K^{2} q^{n} w_{n}^{2}
\end{aligned}
$$

are two correction terms due to the electric field. Here $u \equiv g+\gamma s, K \equiv u \sqrt{(q+1) /(4 \gamma)}$, $z_{i} \equiv-r_{i} /\left(K q^{i / 2}\right)$, and $w_{i} \equiv-\nu_{i} /\left(K q^{i / 2}\right)$. The first correction term was already present in [33] (note, however, the replacement of $\mathcal{E}$ by $\mathcal{E} \cos \varphi$ and of $\alpha, \beta$ by $\alpha^{\prime}, \beta^{\prime}$ ), whereas the second one is new. Both of them are $n$-dependent, negative and increasing (from $-(1 / 2) K^{2} z^{2}$ or $-(1 / 2) K^{2} w^{2}$ to 0$)$ when $n$ goes from 0 to $\infty$.

As in [33], the corresponding eigenvectors can be written in the Bargmann representation associated with some $q$-boson creation and annihilation operators $b^{+}, b$, such that $b b^{+}-q b^{+} b=1$. 
This is based upon the observation that $B^{-}\left(g_{i}, s_{i}, r_{i}, \nu_{i}\right)$ can be expressed as $B^{-}\left(g_{i}, s_{i}, r_{i}, \nu_{i}\right)=$ $(1 / \sqrt{2}) K q^{i / 2}\left(b-t_{i} b^{+}-z_{i}-\mathrm{i} w_{i}\right)$ and that a similar result applies to the adjoint operator $B^{+}\left(g_{i}, s_{i}, r_{i}, \nu_{i}\right)$. The ground-state wavefunction $\psi_{0}(q, t, z, w ; \xi)$, where $\xi \in \mathbb{C}$ represents $b^{+}$, is obtained in the same form as before (see equation (2.59) of [33]), except for the substitution of the complex parameter $z+\mathrm{i} w$ for the real one $z$. The excited-state wavefunctions $\psi_{n}(q, t, z, w ; \xi) \propto P_{n}(q, t, z, w ; \xi) \psi_{0}\left(q, t_{n}, z_{n}, w_{n} ; \xi\right)$ (with $\left.t_{n}=q^{n} t\right)$ undergo a more profound transformation since, on the right-hand side of the recursion relation for the $n$ th-degree polynomials $P_{n}(q, t, z, w ; \xi)$ given in equation (2.69) of [33], $z$ is replaced by $z-\mathrm{i} w$ whereas $z_{n+1}$ is changed into $z_{n+1}+\mathrm{i} w_{n+1}$. For $n=1$ and 2 , for instance, we obtain

$$
\begin{aligned}
& P_{1}(q, t, z, w ; \xi)=\left(1-t^{2} q^{-1}\right)\left[\xi-\left(1-t q^{-1}\right)^{-1} z+\mathrm{i}\left(1+t q^{-1}\right)^{-1} w\right] \\
& P_{2}(q, t, z, w ; \xi)=\left(1-t^{2} q^{-3}\right)\left\{\left(1-t^{2} q^{-1}\right) \xi^{2}-[2]_{q}\left(1-t^{2} q^{-1}\right)\left[\left(1-t q^{-2}\right)^{-1} q^{-1} z\right.\right. \\
& \left.\quad-\mathrm{i}\left(1+t q^{-2}\right)^{-1} q^{-1} w\right]-t+(1-t)\left(1+t q^{-1}\right)\left(1-t q^{-2}\right)^{-2} q^{-1} z^{2} \\
& \left.\quad-(1+t)\left(1-t q^{-1}\right)\left(1+t q^{-2}\right)^{-2} q^{-1} w^{2}-2 \mathrm{i}\left(1-t^{2} q^{-1}\right)\left[\left(1-t q^{-2}\right)\left(1+t q^{-2}\right)\right]^{-1} q^{-1} z w\right\},
\end{aligned}
$$

respectively.

It is worth stressing that in contrast with the energy eigenvalues, which could be derived from those obtained in [32], the corresponding eigenfunctions are an entirely new and nontrivial result (see section 2.4 of [33] for a detailed comparison between our approach and that of [32]).

\section{Function-dependent commutation relation}

\subsection{Commutation relation and uncertainty relation}

Let us consider the commutation relation

$$
[f(X), P]=\mathrm{i}\left[f^{\prime}(X)+\beta P^{2}\right],
$$

where $f(X)$ is some differentiable, real-valued function of a generalized position operator $X$, $f^{\prime}(X)$ denotes its derivative with respect to $X$, and $\beta \in \mathbb{R}^{+}$is some very small parameter (i.e., $\beta \ll 1$ ). For $\beta=0$, equation (3.1) reduces to the relation $[f(x), p]=\mathrm{i} f^{\prime}(x)$, satisfied by the conventional position and momentum operators $x$ and $p=-\mathrm{i} d / d x$, characterized by $[x, p]=\mathrm{i}$. Furthermore, for $\beta \neq 0$, it may be considered as a generalization of Kempf's quadratic commutation relation (2.1) with $\alpha=\kappa=0$ (leading to a nonzero minimal uncertainty $\Delta X_{0}=$ $\sqrt{\beta}$ in position only) since the latter may be retrieved by choosing $f(X)=X$.

For some reasons that will be explained in Section 3.3, we are going to restrict ourselves here to functions $f(X)$ such that

$$
f^{\prime}(X)=a f^{2}(X)+b f(X)+c
$$

for some choice of real constants $a, b$, and $c$. This includes the Kempf's case for which $a=b=0$ and $c=1$. Some other interesting cases to be considered later on are

$$
\begin{aligned}
& f(X)=-e^{-X}, \quad f^{\prime}(X)=e^{-X}, \quad a=0, \quad b=-1, \quad c=0, \\
& f(X)=\tanh X, \quad f^{\prime}(X)=\operatorname{sech}^{2} X, \quad a=-1, \quad b=0, \quad c=1, \\
& f(X)=\tan X, \quad f^{\prime}(X)=\sec ^{2} X, \quad a=1, \quad b=0, \quad c=1 .
\end{aligned}
$$

Observe that in all these examples, $X$ is the lowest-order term in an expansion of $f(X)$ into powers of $X$.

To interpret the generalized commutation relation (3.1) from a physical viewpoint, it is necessary to derive an approximate expression for $[X, P]$. For such a purpose, let us restrict ourselves 
to those states for which $(\Delta X)^{2}=\left\langle\hat{X}^{2}\right\rangle$ (where $\left.\hat{X}=X-\langle X\rangle\right)$ is very small and of the order of $\beta$. On expanding $f(X)$ and $f^{\prime}(X)$ around $\langle X\rangle$ and inserting such expressions in (3.1), we arrive at the relation

$$
\begin{aligned}
{[\hat{X}, P]=} & \mathrm{i}\left(1+\frac{f^{\prime \prime}(\langle X\rangle)}{f^{\prime}(\langle X\rangle)} \hat{X}+\frac{1}{2} \frac{f^{\prime \prime \prime}(\langle X\rangle)}{f^{\prime}(\langle X\rangle)} \hat{X}^{2}+\cdots+\frac{\beta}{f^{\prime}(\langle X\rangle)} P^{2}\right)-\frac{1}{2} \frac{f^{\prime \prime}(\langle X\rangle)}{f^{\prime}(\langle X\rangle)} \\
& \times(\hat{X}[\hat{X}, P]+[\hat{X}, P] \hat{X})-\frac{1}{6} \frac{f^{\prime \prime \prime}(\langle X\rangle)}{f^{\prime}(\langle X\rangle)}\left(\hat{X}^{2}[\hat{X}, P]+\hat{X}[\hat{X}, P] \hat{X}+[\hat{X}, P] \hat{X}^{2}\right)+\cdots
\end{aligned}
$$

Expanding next $[\hat{X}, P]$ into powers of $\hat{X}$,

$$
[\hat{X}, P]=[\hat{X}, P]_{(0)}+[\hat{X}, P]_{(1)} \hat{X}+[\hat{X}, P]_{(2)} \hat{X}^{2}+\cdots,
$$

on both sides of (3.6) and equating successively the $k$ th-order terms in $\hat{X}$ up to $k=2$ leads to the results

$$
[\hat{X}, P]_{(0)}=\mathrm{i}, \quad[\hat{X}, P]_{(1)} \hat{X}=0, \quad[\hat{X}, P]_{(2)} \hat{X}^{2}=\mathrm{i} \frac{\beta}{f^{\prime}(\langle X\rangle)} P^{2},
$$

from which we get the approximate commutation relation

$$
[X, P] \simeq \mathrm{i}\left(1+\frac{\beta}{f^{\prime}(\langle X\rangle)} P^{2}\right)
$$

We conclude that in those states for which $(\Delta X)^{2} \sim \beta$, there is a nonzero minimal uncertainty in $X$, given by $\Delta X_{0} \simeq \sqrt{\beta / f^{\prime}(\langle X\rangle)}$ and therefore dependent on $\langle X\rangle$. For $f(X)=X$, such a dependence disappears so that one retrieves Kempf's result, as it should be.

\subsection{Representations of $X$ and $P$ in terms of conventional position and momentum operators}

In the case of Kempf's quadratic commutation relation corresponding to the choice $f(X)=X$ in (3.1), several different kinds of representations of $X$ and $P$ in terms of conventional operators $x, p$ have been used. Among them, one may quote the momentum representation $P=p$, $X=\left(1+\beta p^{2}\right) x$ (with $\left.x=\mathrm{i} d / d p\right)[9,10,19]$ and the (quasi)position representation $X \simeq x$, $P \simeq p\left(1+(1 / 3) \beta p^{2}\right)$ (with $\left.p=-\mathrm{i} d / d x\right)$ [12]. Whereas the former is exact, the latter is only valid to first order in $\beta$. As we now plan to show, the last one can be extended to the generalized commutation relation (3.1).

Let us indeed look for a representation of (3.1) of the type

$$
X \simeq x, \quad P \simeq p+\beta A(x, p),
$$

where $A(x, p)$ is a so far undetermined function of $x$ and $p$. Inserting this ansatz in (3.1), we find that to first order in $\beta$, the latter is equivalent to $[f(x), A(x, p)]=\mathrm{i} p^{2}$. Such a condition can be easily fulfilled by an operator $A$ such as

$$
A(x, p)=\frac{1}{2}\{\lambda(x), p\}+\frac{1}{2}\left\{\mu(x), p^{3}\right\},
$$

where

$$
\begin{aligned}
& \mu(x)=\frac{1}{3 f^{\prime}} \\
& \lambda(x)=\mu\left[\frac{3}{2}\left(\frac{\mu^{\prime}}{\mu}\right)^{\prime}+\mu\left(\frac{1}{\mu}\right)^{\prime \prime}\right]=\frac{1}{6 f^{\prime}}\left(-\frac{f^{\prime \prime \prime}}{f^{\prime}}+3 \frac{f^{\prime \prime 2}}{f^{\prime 2}}\right),
\end{aligned}
$$


and a prime denotes derivative with respect to $x$. A further simplification occurs for the choice made in (3.2) since equation (3.10) can then be transformed into

$$
\lambda(x)=a+\left(b^{2}-4 a c\right) \mu(x)
$$

with $\mu(x)$ given in $(3.9)$.

The representation (3.7) also allows us to calculate $[X, P]$ to first order in $\beta$. From $[x, A(x, p)]=(\mathrm{i} / 2)\left[2 \lambda(x)+3\left\{\mu(x), p^{2}\right\}\right]$, we indeed obtain

$$
[X, P] \simeq \mathrm{i}\left[1+\beta\left(\lambda(X)+\frac{3}{2}\left\{\mu(X), P^{2}\right\}\right)\right]
$$

after substituting $X$ and $P$ for $x$ and $p$ in the first-order term on the right-hand side.

For the examples considered in (3.3)-(3.5), the representation of $P$ and the commutation relation $[X, P]$ are given by

$$
\begin{aligned}
& P \simeq p+\frac{1}{6} \beta\left\{e^{x}, p+p^{3}\right\}, \\
& {[X, P] \simeq \mathrm{i}\left[1+\beta\left(\frac{1}{3} e^{X}+\frac{1}{2}\left\{e^{X}, P^{2}\right\}\right)\right],} \\
& P \simeq p+\beta\left(-p+\frac{1}{6}\left\{\cosh ^{2} x, 4 p+p^{3}\right\}\right), \\
& {[X, P] \simeq \mathrm{i}\left[1+\beta\left(-1+\frac{4}{3} \cosh ^{2} X+\frac{1}{2}\left\{\cosh ^{2} X, P^{2}\right\}\right)\right],} \\
& P \simeq p+\beta\left(p+\frac{1}{6}\left\{\cos ^{2} x,-4 p+p^{3}\right\}\right), \\
& {[X, P] \simeq \mathrm{i}\left[1+\beta\left(1-\frac{4}{3} \cos ^{2} X+\frac{1}{2}\left\{\cos ^{2} X, P^{2}\right\}\right)\right],}
\end{aligned}
$$

respectively.

For the special case of (3.3), it is worth mentioning the existence of an alternative exact representation of $f(X)$ and $P$,

$$
-e^{-X}=-e^{-x}+\beta p^{2}, \quad P=p .
$$

In view of the applications to quantum mechanics to be carried out in the next subsection, it is worth inquiring into the restrictions, if any, that the Hermiticity of the deformed operators $X$ and $P$ imposes on square-integrable functions. For representation (3.7), let us consider two functions $\psi(x), \phi(x) \in L^{2}\left(x_{1}, x_{2}\right)$ and expand them to first order in $\beta$ as $\psi(x)=\psi^{0}(x)+\beta \Delta \psi(x)$, $\phi(x)=\phi^{0}(x)+\beta \Delta \phi(x)$. Then, in the same approximation, the Hermiticity of $P$ in $L^{2}\left(x_{1}, x_{2}\right)$ is equivalent to the condition

$$
\int_{x_{1}}^{x_{2}} d x \psi^{0 *}(x) A(x, p) \phi^{0}(x)=\left[\int_{x_{1}}^{x_{2}} d x \phi^{0 *}(x) A(x, p) \psi^{0}(x)\right]^{*} .
$$

On using (3.8) and integrating several times by parts, it is easy to show that equation (3.15) amounts to the two restrictions

$$
\begin{aligned}
& \lambda\left|\psi^{0}\right|^{2} \rightarrow 0 \quad \text { for } x \rightarrow x_{1} \text { and } x \rightarrow x_{2}, \\
& 2 \mu^{\prime \prime}\left|\psi^{0}\right|^{2}+\mu^{\prime}\left(\psi^{0 *} \psi^{0 \prime}+\psi^{0 / *} \psi^{0}\right)+2 \mu\left(\psi^{0 *} \psi^{0 \prime \prime}-\left|\psi^{0 / *}\right|^{2}+\psi^{0 / \prime *} \psi^{0}\right) \rightarrow 0 \\
& \quad \text { for } x \rightarrow x_{1} \text { and } x \rightarrow x_{2},
\end{aligned}
$$

in the special case where $\phi^{0}(x)=\psi^{0}(x)$. In contrast, for representation (3.14), no further condition is found to ensure the Hermiticity of $e^{-X}$. 


\subsection{Applications to quantum mechanics}

Let us a consider a Hamiltonian

$$
H=\frac{1}{2} P^{2}+V(X)
$$

where $X$ and $P$ satisfy the function-dependent commutation relation (3.1) for some choice of $f(X)$. To be able to solve the eigenvalue problem for such a Hamiltonian, we shall impose that (i) it is factorizable as shown in (2.14), where the operators $B^{ \pm}$, now depending only on three real parameters $g, s, r$, are chosen in the form

$$
B^{ \pm}(g, s, r)=\frac{1}{\sqrt{2}}[\mp \mathrm{i} g P+s f(X)+r],
$$

and (ii) $H$ is the first member $H_{0}$ of a hierarchy of Hamiltonians $H_{i}, i=0,1,2, \ldots$, similar to (3.18) and satisfying a SI condition of type (2.16).

The factorization of $H$ implies that there exists a close connection between $f(X)$ and $V(X)$, while the SI condition leads to equation (3.2), expressing $f^{\prime}(X)$ as a second-degree polynomial in $f(X)$. The proof of these assertions relies on the relation

$$
B^{ \pm}(g, s, r) B^{\mp}(g, s, r)=\frac{1}{2}\left\{g(g \mp \beta s) P^{2}+[s f(X)+r]^{2} \mp g s f^{\prime}(X)\right\}
$$

resulting from (3.1) and (3.19). On comparing (3.18) with (3.20), it is indeed obvious that $V(X)$ can be written in terms of $f(X)$ and $f^{\prime}(X)$. On the other hand, since we need four equations (but not more) to determine the four types of parameters $g_{i}, s_{i}, r_{i}$, and $\epsilon_{i}$ from the SI condition, it is evident that $f^{\prime}(X)$ should contain the same kind of terms as $[s f(X)+r]^{2}$, which is achieved by assuming condition (3.2) (see [36] for a systematic use of such a type of reasoning). As a result, the precise relationship between $V(X)$ and $f(X)$ reads

$$
V(X)=\frac{1}{2}\left[s(s-a g) f^{2}(X)+s(2 r-b g) f(X)+r^{2}-c g s\right]+\epsilon_{0} .
$$

Observe that in Kempf's case, $f(X)=X$ leads to a harmonic oscillator potential [23].

When using the representation (3.7) of $X$ and $P$ valid to first order in $\beta$, the Hamiltonian (3.18) can be written in the same approximation as

$$
H \simeq \frac{1}{2} p\left[1+\frac{1}{6} \beta\left(2 a-\frac{b^{2}-4 a c}{f^{\prime}(x)}\right)\right] p+\beta p^{2} \frac{1}{3 f^{\prime}(x)} p^{2}+V(x)
$$

in terms of the operators $x, p$. As compared with conventional Hamiltonians, there is a small additional term proportional to $p^{4}$ as in Kempf's case [12], but for $f(X) \neq X$ we also observe a position dependence of the terms in $p^{2}$ and $p^{4}$. Furthermore, bound-state wavefunctions of $H$ have not only to be square integrable on the (finite or infinite) interval of definition $\left(x_{1}, x_{2}\right)$ of $V(x)$, but also to satisfy some additional restrictions ensuring the Hermiticity of $H$. For such a purpose, it is enough to impose that $P$ be Hermitian, hence that conditions (3.16) and (3.17) be fulfilled.

We shall now proceed to consider several examples associated with the functions (3.3)-(3.5).

\subsubsection{Pöschl-Teller potentials}

For $f(X)=\tanh X$ corresponding to (3.4), we get the one-parameter hyperbolic Pöschl-Teller potential

$$
V(X)=-\frac{1}{2} A(A+1) \operatorname{sech}^{2} X, \quad A \geq 1,
$$


by choosing

$$
s=\sqrt{\frac{A(A+1)}{1+k}}, \quad g=k s, \quad r=0, \quad \epsilon_{0}=-\frac{1}{2} s^{2}
$$

in (3.21). Here $k$ is defined by

$$
k=\frac{1+\beta A(A+1)+\Delta}{2 A(A+1)}, \quad \Delta \equiv \sqrt{[1+\beta A(A+1)]^{2}+4 A(A+1)} .
$$

In the $\beta \rightarrow 0$ limit, equations (3.22) and (3.23) lead to the conventional result $s \rightarrow A, g \rightarrow 1$, so that $B^{ \pm}(g, s) \rightarrow B^{ \pm}(A)=(\mp \mathrm{i} p+A \tanh x) / \sqrt{2}[24]$.

The SI condition is then solved by considering the combinations of parameters

$$
u_{i}=g_{i}+\mathrm{i} \sqrt{\beta} s_{i}=\left|u_{i}\right| e^{\mathrm{i} \varphi_{i}} .
$$

The results read

$$
\left|u_{i}\right|=|u|, \quad \varphi_{i}=\varphi+\frac{\mathrm{i}}{2} \phi, \quad r_{i}=0, \quad \epsilon_{i+1}=\frac{1}{2}\left(s_{i}^{2}-s_{i+1}^{2}\right),
$$

where it follows from (3.22) that

$$
|u|=\sqrt{1+\beta A(A+1)}, \quad \cos \varphi=\frac{g}{\sqrt{1+\beta A(A+1)}}, \quad \sin \varphi=\frac{\sqrt{\beta} s}{\sqrt{1+\beta A(A+1)}},
$$

and $\phi$ is defined by

$$
e^{\mathrm{i} \phi}=\frac{1-\mathrm{i} \sqrt{\beta}}{1+\mathrm{i} \sqrt{\beta}}, \quad \cos \phi=\frac{1-\beta}{1+\beta}, \quad \sin \phi=-\frac{2 \sqrt{\beta}}{1+\beta} .
$$

The energy eigenvalues of the (generalized) hyperbolic Pöschl-Teller Hamiltonian can be expressed as

$$
E_{n}=\sum_{i=0}^{n} \epsilon_{i}=-\frac{1}{2} s_{n}^{2}=-\frac{|u|^{2}}{2 \beta} \sin ^{2}\left(\varphi+\frac{n}{2} \phi\right)
$$

On expanding them into powers of $\beta$ and keeping only the first two terms, we obtain

$$
\begin{aligned}
& E_{n} \simeq-\frac{1}{2}(A-n)^{2}\left(1-\beta \delta_{n}+\cdots\right), \\
& \delta_{n}=\frac{1}{(2 A+1)(A-n)}\left\{A^{2}+n\left[(n+1) A-\frac{n^{2}+2}{3}\right](2 A+1)\right\} .
\end{aligned}
$$

In the $\beta \rightarrow 0$ limit, we get back the bound-state spectrum of the conventional Pöschl-Teller Hamiltonian, $E_{n}^{0}=-(1 / 2)(A-n)^{2}, n=0,1, \ldots, n_{\max }, A-1 \leq n_{\max }<A$ [24], as it should be. It can be easily shown that for $\beta \neq 0$, the first-order relative correction in (3.26) has the opposite sign and increases with $n$ : $0<\delta_{0}<\delta_{1}<\cdots<\delta_{n_{\max }}$. For the approximation (3.26) to be meaningful, we must therefore restrict ourselves to $\beta$ values such that $\beta \ll\left(\delta_{n_{\max }}\right)^{-1}$, which for integer $A$, for instance, gives rise to the condition $\beta \ll 3(2 A+1)\left(4 A^{4}+2 A^{3}-7 A^{2}+A+3\right)^{-1}$.

We can get the corresponding eigenfunctions

$$
\psi_{n}(g, s ; x) \simeq \psi_{n}^{0}(A ; x)+\beta \Delta \psi_{n}(g, s ; x)
$$


in the representation (3.12), wherein the (generalized) Pöschl-Teller Hamiltonian and the operators $B^{ \pm}(g, s)$ take the form

$$
\begin{aligned}
& H \simeq \frac{1}{2} p\left[1-\frac{1}{3} \beta\left(1+2 \cosh ^{2} x\right)\right] p+\frac{1}{3} \beta p^{2} \cosh ^{2} x p^{2}-\frac{1}{2} A(A+1) \operatorname{sech}^{2} x, \\
& B^{ \pm}(g, s) \simeq B_{0}^{ \pm}(A)+\beta \Delta B^{ \pm}(g, s), \\
& \Delta B^{ \pm}(g, s)=\frac{1}{\sqrt{2}}\left[\mp \frac{\mathrm{i}}{2}(A-2) p \mp \frac{\mathrm{i}}{6}\left\{\cosh ^{2} x, 4 p+p^{3}\right\}-\frac{A^{2}}{2(2 A+1)} \tanh x\right]
\end{aligned}
$$

with $p=-\mathrm{i} d / d x$.

Since the ground state wavefunction $\psi_{0}(g, s ; x)$ must be annihilated by the operator $B^{-}(g, s)$, the first-order correction term $\Delta \psi_{0}(g, s ; x)$ with respect to $\psi_{0}^{0}(A ; x)=N_{0}(A) \operatorname{sech}^{A} x$ (where $\left.N_{0}(A)=\{\Gamma(A+1 / 2) /[\Gamma(1 / 2) \Gamma(A)]\}^{1 / 2}\right)$ satisfies the first-order differential equation

$$
B_{0}^{-}(A) \Delta \psi_{0}(g, s ; x)=-\Delta B^{-}(g, s) \psi_{0}^{0}(A ; x) .
$$

A straightforward calculation leads to

$$
\Delta \psi_{0}(g, s ; x)=N_{0}(A)\left[-\frac{1}{6} A(A-1)(A-2) \operatorname{sech}^{A-2} x+C \operatorname{sech}^{A} x \ln \cosh x+D \operatorname{sech}^{A} x\right],
$$

with $C \equiv A(A+1)\left(2 A^{2}+2 A-1\right) /[3(2 A+1)]$, while $D$ is some integration constant. The latter can be determined from the normalization condition of $\psi_{0}(g, s ; x)$ to first order in $\beta$. In the special case where $A$ is integer, it is given by

$$
D=\frac{1}{12} A(A-1)(2 A-1)+C\left(\ln 2+\sum_{j=1}^{2 A-1} \frac{(-1)^{j}}{j}\right) .
$$

Finally, it can be easily shown that for $\psi_{0}(g, s ; x)$, the conditions (3.16) and (3.17) imposed by the Hermiticity of $P$ (hence of $H$ ) amount to the restriction $\operatorname{sech}^{2 A-2} x \rightarrow 0$ for $x \rightarrow \pm \infty$. The allowed $A$ values are therefore $A>1$ (excluding $A=1$ ).

The excited-state wavefunctions can be calculated in a similar way. For instance, the firstorder correction $\Delta \psi_{1}(g, s ; x)$ to the first-excited state wavefunction can be obtained from

$$
\Delta \psi_{1}(g, s ; x) \propto B_{0}^{+}(A) \Delta \psi_{0}\left(g_{1}, s_{1} ; x\right)+\Delta B^{+}(g, s) \psi_{0}^{0}(A-1 ; x),
$$

where $g_{1}$ and $s_{1}$ are given by equations (3.24)-(3.25).

The treatment of the one-parameter trigonometric Pöschl-Teller potential

$$
V(X)=\frac{1}{2} A(A-1) \sec ^{2} X, \quad-\frac{\pi}{2} \leq X \leq \frac{\pi}{2}, \quad A>1,
$$

is entirely analogous. With $f(X)=\tan X$ corresponding to (3.5) and (3.13), we are led to the energy spectrum

$$
\begin{aligned}
& E_{n} \simeq \frac{1}{2}(A+n)^{2}\left(1+\beta \delta_{n}+\cdots\right) \\
& \delta_{n}=\frac{1}{(2 A-1)(A+n)}\left\{A^{2}+n\left[(n+1) A+\frac{n^{2}+2}{3}\right](2 A-1)\right\},
\end{aligned}
$$

valid up to first order in $\beta$. As previously, the correction terms satisfy the property $0<\delta_{0}<$ $\delta_{1}<\cdots<\delta_{n}<\cdots$. 


\subsubsection{Morse potential}

The Morse potential

$$
V(X)=\frac{1}{2} B^{2} e^{-2 X}-\frac{1}{2} B(2 A+1) e^{-X}, \quad A, B>0,
$$

is associated with the choice $f(X)=-e^{-X}$ in (3.3). In such a case, it is straightforward to show that

$$
\begin{aligned}
& s=B, \quad g=\frac{1}{2} \beta B+\Delta, \quad r=A+\frac{1}{2}-\frac{g}{2}, \quad \Delta \equiv \sqrt{1+\frac{1}{4} \beta^{2} B^{2}}, \\
& s_{i}=s, \quad g_{i}=g+i \beta B, \quad r_{i}=r-i g-\frac{1}{2} i^{2} \beta B,
\end{aligned}
$$

and

$$
E_{n}=-\frac{1}{2} r_{n}^{2}=-\frac{1}{2}\left[A+\frac{1}{2}-\frac{1}{4}\left(2 n^{2}+2 n+1\right) \beta B-\left(n+\frac{1}{2}\right) \Delta\right]^{2},
$$

where in the $\beta \rightarrow 0$ limit, we obtain the conventional energy spectrum $E_{n}^{0}=-(1 / 2)(A-n)^{2}$, $n=0,1, \ldots, n_{\max }, A-1 \leq n_{\max }<A[24]$.

First-order corrections to the corresponding wavefunctions can be obtained by using the representation (3.11) as for Pöschl-Teller potentials. We shall instead use here the alternative representation (3.14) because it leads to exact results for the Hamiltonian

$$
H=\frac{1}{2} p\left[1+\beta B\left(2 A+1-2 B e^{-x}\right)\right] p+\frac{1}{2} \beta^{2} B^{2} p^{4}+\frac{1}{2} B^{2} e^{-2 x}-\frac{1}{2} B(2 A+1-\beta B) e^{-x} .
$$

As observed at the end of Section 3.2, bound-state wavefunctions of $H$ do not have to satisfy any extra condition apart from square integrability on the real line.

As shown in the appendix, by employing the properties of the operators

$$
B^{ \pm}(g, B, r)=\frac{1}{\sqrt{2}}\left(-\beta B \frac{d^{2}}{d x^{2}} \mp g \frac{d}{d x}-B e^{-x}+r\right),
$$

the ground- and excited-state wavefunctions of such a Hamiltonian, corresponding to the energy eigenvalues (3.31), can be expressed in terms of Bessel functions as

$$
\psi_{0}(g, B, r ; x) \propto \exp \left(\frac{g x}{2 \beta B}\right) J_{\nu}\left(\frac{2}{\sqrt{\beta}} e^{-x / 2}\right)
$$

and

$$
\psi_{n}(g, B, r ; x) \propto \sum_{j=0}^{n} c_{j} \exp \left(\frac{g_{n-j} x}{2 \beta B}\right) J_{\nu_{n}+j}\left(\frac{2}{\sqrt{\beta}} e^{-x / 2}\right),
$$

respectively. Here $\nu$ is defined by

$$
\nu=\frac{1}{\beta B} \sqrt{g^{2}+4 \beta B r}
$$

and a similar expression applies to $\nu_{n}$ in terms of $g_{n}$ and $r_{n}$, while $c_{j}$ denote some constants.

From the properties of Bessel functions [37], it follows that for $x \rightarrow-\infty, \psi_{0}(g, B, r ; x)$ decays exponentially as $\exp [-(g /(2 \beta B)+1 / 4)|x|]$, while for $x \rightarrow \infty$, it behaves as $\exp [(g /(2 \beta B)-\nu / 2) x]$. It is therefore normalizable on the real line provided $\nu>g /(\beta B)$ or, in other words, $r>0$. From the expression of $r$ given in (3.30), we conclude that the Hamiltonian (3.32) has at least one 
bound state, given by (3.34), if $\beta$ takes a value satisfying the inequality $\beta<4 A(A+1)[(2 A+$ 1) $B]^{-1}$. Whenever such a condition is satisfied, there may actually also exist excited states. Since the normalizability condition of $\psi_{n}(g, B, r ; x)$, given in (3.35), is $r_{n}>0$, the allowed values of $n$ are $n=0,1, \ldots, n_{\max }$, where $n_{\max }$ is the largest integer for which $r_{n}>0$, i.e., the integer fulfilling the condition

$$
\frac{1}{\beta B}\left(-\frac{3}{2} \beta B-\Delta+\sqrt{1+\beta B(2 A+1)}\right) \leq n_{\max }<\frac{1}{\beta B}\left(-\frac{1}{2} \beta B-\Delta+\sqrt{1+\beta B(2 A+1)}\right) .
$$

Observe that for $\beta \rightarrow 0$, equation (3.37) leads to the conventional result $A-1 \leq n_{\max }<A$, as it should be.

\section{Final remarks}

In the present paper, we have considered two extensions of Kempf's quadratic canonical commutation relation in one dimension. For both of them, we have studied how they alter the minimal uncertainties in position and/or momentum and we have proposed some applications to quantum mechanics.

The first one is the most general quadratic commutation relation, which turns out to also include a $q$-deformed Heisenberg algebra as a special case. Although it can be reduced to Kempf's commutation relation by applying some transformations to the position and momentum operators, we have shown that it gives rise to more general expressions for the nonzero minimal uncertainties in position and momentum and that it has a definite influence on the energy spectrum and the corresponding eigenfunctions of the harmonic oscillator in an electric field, first considered in [33].

In the second generalization, the position operator $X$ has been replaced in the simplest quadratic commutation relation (with only a nonzero minimal uncertainty in position) by some function $f(X)$ in such a way that the choice $f(X)=X$ gives back the original commutation relation. From the uncertainty point of view, there appears a new and interesting effect in the sense that the minimal uncertainty in $X$ now becomes dependent on the average position $\langle X\rangle$ through the function $f^{\prime}(\langle X\rangle)$.

Next, to any function-dependent commutation relation of this type, such that $f^{\prime}(X)$ satisfies equation (3.2), we have associated a definite family of potentials $V(X)$, whose spectrum can be exactly determined through SUSYQM and SI techniques in the same way as we had done for the harmonic oscillator in the case of the original choice $f(X)=X$ [23]. Along these lines, we have treated in detail the cases of the one-parameter hyperbolic and trigonometric Pöschl-Teller potentials, as well as that of the Morse potential.

Furthermore, for any $f(X)$ we have obtained a representation of our deformed algebra in terms of conventional position and momentum operators $x, p$, up to first order in the deformation parameter $\beta$. This has allowed us to write the corresponding Hamiltonian in terms of $x, p$, and to determine its bound-state wavefunctions in the same approximation. Finally, for the Morse potential, the use of an alternative exact representation of the associated algebra has led us to some exact results for the Hamiltonian and its bound-state wavefunctions.

In both types of representations, the resulting Hamiltonians are of fourth order in $p$ and, in addition, the $p$-dependent terms may also depend on $x$. The former property had already been observed elsewhere [12] in the context of Kempf's quadratic commutation relations, while the latter reminds one of the well-known equivalence between some deformed commutation relations and position-dependent masses (PDM) [38].

Both characteristics may be very interesting in the area of condensed-matter problems. A dependence of the kinetic energy on the position is indeed often used there due to its relevance in describing the dynamics of electrons in compositionally-graded crystals [39], quantum dots [40] 
and liquid crystals [41], for instance. Furthermore, it has been shown that fourth-order terms in the momentum may depict nonparabolicity effects in quantum wells [42].

Some applications may arise in other fields too. Let us mention the occurrence of PDM in the energy-density functional approach to the quantum many-body problem in the context of nonlocal terms of the accompanying potential with applications to nuclei [43], quantum liquids [44] and metal clusters [45]. It is also worth noting that higher-order terms in $p^{2}$ appear in semiclassical approaches to the Klein-Gordon equation, as well as in Hermitian Hamiltonians equivalent to $\mathcal{P} \mathcal{T}$-symmetric or, more generally, pseudo-Hermitian ones [46, 47, 48].

In conclusion, we would like to stress the relevance of the innovative deformed-algebra approach proposed here to derive exact or approximate solutions to Schrödinger equations containing generalized Hamiltonians such as those given in (3.27) and (3.32). We do think that it will open a new inspiration for future study.

\section{Appendix. Bound-state wavefunctions of the generalized Morse Hamiltonian (3.32)}

To calculate the bound-state wavefunctions $\psi_{n}(g, B, r ; x)$ of the Hamiltonian (3.32), it proves convenient to introduce two auxiliary variables

$$
y=B e^{-x}, \quad z=2 \sqrt{\frac{y}{\beta B}},
$$

both varying on the half line $(0, \infty)$, and to define

$$
\psi_{n}(g, B, r ; x)=\phi_{n}(g, B, r ; y)=\chi_{n}(g, B, r ; z),
$$

satisfying the normalization condition

$$
\int_{-\infty}^{+\infty} d x\left|\psi_{n}(g, B, r ; x)\right|^{2}=\int_{0}^{+\infty} \frac{d y}{y}\left|\phi_{n}(g, B, r ; y)\right|^{2}=2 \int_{0}^{+\infty} \frac{d z}{z}\left|\chi_{n}(g, B, r ; z)\right|^{2}=1 .
$$

The operators $B^{ \pm}(g, B, r)$ in equation (3.33) can then be written as

$$
B^{ \pm}(g, B, r)=\frac{1}{\sqrt{2}}\left[-\beta B\left(y \frac{d}{d y}\right)^{2} \pm g y \frac{d}{d y}-y+r\right] .
$$

The ground-state wavefunction being a zero mode of $B^{-}(g, B, r)$ is the solution of the secondorder differential equation

$$
\left[\beta B y^{2} \frac{d^{2}}{d y^{2}}+(g+\beta B) y \frac{d}{d y}+y-r\right] \phi_{0}(g, B, r ; y)=0
$$

satisfying condition (A.3). Since $y=0$ is a regular singular point of this equation, we may look for a solution of the type

$$
\phi_{0}(g, B, r ; y)=y^{\rho} \sum_{j=0}^{\infty} a_{j} y^{j}
$$

where $a_{j}$ are some constants, $a_{0} \neq 0$ and $\rho>0$ in order to fulfil (A.3). Inserting (A.5) in (A.4), we easily find that $\rho=(1 / 2)(-g /(\beta B)+\nu)$ (with $\nu$ defined in $(3.36)$ ), provided the condition $r>0$ is satisfied. Furthermore

$$
\sum_{j=0}^{\infty} a_{j} y^{j} \propto{ }_{0} F_{1}\left(\nu+1 ;-\frac{y}{\beta B}\right) \propto y^{-\nu / 2} J_{\nu}(z),
$$

where in the last step use is made of equation (9.1.69) of [37]. This proves equation (3.34). 
The first-excited state wavefunction can be obtained by acting with $B^{+}(g, B, r)$ on $\psi_{0}\left(g_{1}, B, r_{1} ; x\right)$. On using the analogue of equation (A.4) for $\phi_{0}\left(g_{1}, B, r_{1} ; y\right)$ to eliminate the second-order derivative with respect to $y$, it can be shown that

$$
\phi_{1}(g, B, r ; y) \propto\left[\left(g+g_{1}\right) y \frac{d}{d y}+r-r_{1}\right] \phi_{0}\left(g_{1}, B, r_{1} ; y\right) .
$$

Since from (3.30) it results that $r-r_{1}=(1 / 2)\left(g+g_{1}\right)$, this equation reduces to

$$
\chi_{1}(g, B, r ; z) \propto\left(z \frac{d}{d z}+1\right) \chi_{0}\left(g_{1}, B, r_{1} ; z\right) .
$$

Equation (9.1.29) of [37] then leads to

$$
\chi_{1}(g, B, r ; z) \propto-z^{-g /(\beta B)} J_{\nu_{1}+1}(z)+\left(2 \rho_{1}+1\right) z^{-g_{1} /(\beta B)} J_{\nu_{1}}(z)
$$

with $\rho_{1}=(1 / 2)\left(-g_{1} /(\beta B)+\nu_{1}\right)$. Combining this result with (A.1) and (A.2) finally yields equation (3.35) for $n=1$.

The proof of such an equation for higher $n$ values is based upon observing that

$$
\phi_{n}(g, B, r ; y) \propto\left\{\prod_{j=0}^{n-1}\left[\left(g_{j}+g_{n}\right)\left(y \frac{d}{d y}-j\right)+r_{j}-r_{n}\right]\right\} \phi_{0}\left(g_{n}, B, r_{n} ; y\right)
$$

and applying $n$ times equation (9.1.29) of [37]. Equation (A.6) itself can be demonstrated by induction over $n$ by starting from the relation $\phi_{n}(g, B, r ; y) \propto B^{+}(g, B, r) \phi_{n-1}\left(g_{1}, B, r_{1} ; y\right)$, commuting $B^{+}(g, B, r)$ with the factors on its right until it acts on $\phi_{0}\left(g_{n}, B, r_{n} ; y\right)$ and eliminating the second-order derivative as done above.

\section{Acknowledgements}

V.M.T. thanks the National Fund for Scientific Research (FNRS), Belgium, for financial support.

\section{References}

[1] Gross D.J., Mende P.F., String theory beyond the Planck scale, Nuclear Phys. B 303 (1988), $407-454$.

[2] Amati D., Ciafaloni M., Veneziano G., Can spacetime be probed below the string size?, Phys. Lett. B 216 (1989), 41-47.

[3] Maggiore M., The algebraic structure of the generalized uncertainty principle, Phys. Lett. B 319 (1993), 83-86, hep-th/9309034.

[4] Connes A., Gravity coupled with matter and the foundation of non-commutative geometry, Comm. Math. Phys. 182 (1996), 155-176, hep-th/9603053.

[5] Amelino-Camelia G., Mavromatos N.E., Ellis J., Nanopoulos D.V., On the space-time uncertainty relations of Liouville strings and D-branes, Modern Phys. Lett. A 12 (1997), 2029-2036, hep-th/9701144.

[6] Seiberg N., Witten E., String theory and noncommutative geometry, JHEP 9909 (1999), 032, 93 pages, hep-th/9908142.

[7] Kempf A., Uncertainty relation in quantum mechanics with quantum group symmetry, J. Math. Phys. 35 (1994), 4483-4496, hep-th/9311147.

[8] Hinrichsen H., Kempf A., Maximal localization in the presence of minimal uncertainties in positions and in momenta, J. Math. Phys. 37 (1996), 2121-2137, hep-th/9510144.

[9] Kempf A., Mangano G., Mann R.B., Hilbert space representation of the minimal length uncertainty relation, Phys. Rev. D 52 (1995), 1108-1118, hep-th/9412167.

[10] Kempf A., Non-pointlike particles in harmonic oscillators, J. Phys. A: Math. Gen. 30 (1997), 2093-2102, hep-th/9604045. 
[11] Kempf A., Quantum field theory with nonzero minimal uncertainties in position and momentum, hep-th/9405067.

[12] Brau F., Minimal length uncertainty relation and the hydrogen atom, J. Phys. A: Math. Gen. 32 (1999), 7691-7696, quant-ph/9905033.

[13] Benczik S., Chang L.N., Minic D., Takeuchi T., Hydrogen-atom spectrum under a minimal-length hypothesis, Phys. Rev. A 72 (2005), 012104, 4 pages, hep-th/0502222.

[14] Stetsko M.M., Tkachuk V.M., Perturbation hydrogen-atom spectrum in deformed space with minimal length, Phys. Rev. A $\mathbf{7 4}$ (2006), 012101, 5 pages.

[15] Brau F., Buisseret F., Minimal length uncertainty relation and gravitational quantum well, Phys. Rev. D 74 (2006), 036002, 5 pages, hep-th/0605183.

[16] Nouicer K., An exact solution of the one-dimensional Dirac oscillator in the presence of minimal lengths, J. Phys. A: Math. Gen. 39 (2006), 5125-5134.

[17] Chang L.N., Minic D., Okamura N., Takeuchi T., Effect of the minimal length uncertainty relation on the density of states and the cosmological constant problem, Phys. Rev. D 65 (2002), 125028, 7 pages, hep-th/0201017.

[18] Benczik S., Chang L.N., Minic D., Okamura N., Rayyan S., Takeuchi T., Short distance versus long distance physics: The classical limit of the minimal length uncertainty relation, Phys. Rev. D 66 (2002), 026003, 11 pages, hep-th/0204049.

[19] Chang L.N., Minic D., Okamura N., Takeuchi T., Exact solution of the harmonic oscillator in arbitrary dimensions with minimal length uncertainty relations, Phys. Rev. D 65 (2002), 125027, 8 pages, hep-th/0111181.

[20] Fityo T.V., Vakarchuk I.O., Tkachuk V.M., One-dimensional Coulomb-like problem in deformed space with minimal length, J. Phys. A: Math. Gen. 39 (2006), 2143-2150, quant-ph/0507117.

[21] Fityo T.V., Vakarchuk I.O., Tkachuk V.M., WKB approximation in deformed space with minimal length, J. Phys. A: Math. Gen. 39 (2006), 379-388, quant-ph/0510018.

[22] Kempf A., Quantum group symmetric Bargmann-Fock space: integral kernels, Green functions, driving forces, J. Math. Phys. 34 (1993), 969-987.

[23] Quesne C., Tkachuk V.M., Harmonic oscillator with nonzero minimal uncertainties in both position and momentum in a SUSYQM framework, J. Phys. A: Math. Gen. 36 (2003), 10373-10390, math-ph/0306047.

[24] Cooper F., Khare A., Sukhatme U., Supersymmetry and quantum mechanics, Phys. Rep. 251 (1995), 267385, hep-th/9405029.

[25] Gendenshtein L.E., Derivation of exact spectra of the Schrödinger equation by means of supersymmetry, JETP Lett. 38 (1983), 356-359.

[26] Dabrowska J., Khare A., Sukhatme U., Explicit wavefunctions for shape-invariant potentials by operator techniques, J. Phys. A: Math. Gen. 21 (1988), L195-L200.

[27] Spiridonov V., Exactly solvable potentials and quantum algebras, Phys. Rev. Lett. 69 (1992), 398-401, hep-th/9112075.

[28] Spiridonov V., Deformed conformal and supersymmetric quantum mechanics, Modern Phys. Lett. A 7 (1992), 1241-1252, hep-th/9202013.

[29] Khare A., Sukhatme U.P., New shape-invariant potentials in supersymmetric quantum mechanics, J. Phys. A: Math. Gen. 26 (1993), L901-L904, hep-th/9212147.

[30] Barclay D.T., Dutt R., Gangopadhyaya A., Khare A., Pagnamenta A., Sukhatme U., New exactly solvable Hamiltonians: shape invariance and self-similarity, Phys. Rev. A 48 (1993), 2786-2797, hep-ph/9304313.

[31] Lutzenko I., Spiridonov V., Zhedanov A., On the spectrum of a $q$-oscillator with a linear interaction, Phys. Lett. A 204 (1995), 236-242.

[32] Loutsenko I., Spiridonov V., Vinet L., Zhedanov A., Spectral analysis of $q$-oscillator with general bilinear interaction, J. Phys. A: Math. Gen. 31 (1998), 9081-9094.

[33] Quesne C., Tkachuk V.M., More on a SUSYQM approach to the harmonic oscillator with nonzero minimal uncertainties in position and/or momentum, J. Phys. A: Math. Gen. 37 (2004), 10095-10114, math-ph/0312029.

[34] Quesne C., Tkachuk V.M., Dirac oscillator with nonzero minimal uncertainty in position, J. Phys. A: Math. Gen. 38 (2005), 1747-1766, math-ph/0412052. 
[35] Pillin M., On the deformability of Heisenberg algebras, Comm. Math. Phys. 180 (1996), 23-38, q-alg/9508014.

[36] Bagchi B., Banerjee A., Quesne C., Tkachuk V.M., Deformed shape invariance and exactly solvable Hamiltonians with position-dependent effective mass, J. Phys. A: Math. Gen. 38 (2005), 2929-2946, quant-ph/0412016.

[37] Abramowitz M., Stegun I.A., Handbook of mathematical functions, Dover, New York, 1965.

[38] Quesne C., Tkachuk V.M., Deformed algebras, position-dependent effective masses and curved spaces: an exactly solvable Coulomb problem, J. Phys. A: Math. Gen. 37 (2004), 4267-4282, math-ph/0403047.

[39] Geller M.R., Kohn W., Quantum mechanics of electrons in crystals with graded composition, Phys. Rev. Lett. 70 (1993), 3103-3106.

[40] Serra Ll., Lipparini E., Spin response of unpolarized quantum dots, Europhys. Lett. 40 (1997), 667-672.

[41] Barranco M., Pi M., Gatica S.M., Hernández E.S., Navarro J., Structure and energetics of mixed ${ }^{4} \mathrm{He}^{3}{ }^{3} \mathrm{He}$ drops, Phys. Rev. B 56 (1997), 8997-9003.

[42] Ekenberg U., Nonparabolicity effects in a quantum well: sublevel shift, parallel mass, and Landau levels, Phys. Rev. B 40 (1989), 7714-7726.

[43] Ring P., Schuck P., The nuclear many body problem, Springer, New York, 1980.

[44] Arias de Saavedra F., Boronat J., Polls A., Fabrocini A., Effective mass of one ${ }^{4}$ He atom in liquid ${ }^{3}$ He, Phys. Rev. B 50 (1994), 4248-4251.

[45] Puente A., Serra Ll., Casas M., Dipole excitation of Na clusters with a non-local energy density functional, Z. Phys. D 31 (1994), 283-286.

[46] Jones H.F., On pseudo-Hermitian Hamiltonians and their Hermitian counterparts, J. Phys. A: Math. Gen. 38 (2005), 1741-1746.

[47] Mostafazadeh A., $\mathcal{P} \mathcal{T}$-symmetric cubic anharmonic oscillator as a physical model, J. Phys. A: Math. Gen. 38 (2005), 6557-6570, Corrigendum, J. Phys. A: Math. Gen. 38 (2005), 8185-8185, quant-ph/0411137.

[48] Bagchi B., Quesne C., Roychoudhury R., Pseudo-Hermitian versus Hermitian position-dependentmass Hamiltonians in a perturbative framework, J. Phys. A: Math. Gen. 39 (2006), L127-L134, quant-ph/0511182. 